\title{
A novel nonlive, adjuvanted herpes zoster subunit vaccine: a report on the emerging clinical data and safety profile
}

\author{
Federica Brosio' \\ Giulia Masetti' \\ Giulio Matteo' \\ Armando Stefanati ${ }^{2}$ \\ Giovanni Gabutti ${ }^{2}$ \\ 'Postgraduate School of Hygiene and \\ Preventive Medicine, Department \\ of Medical Sciences, University of \\ Ferrara, Ferrara, Italy; ${ }^{2}$ Department \\ of Medical Sciences, University of \\ Ferrara, Ferrara, Italy
}

This article was published in the following Dove Press journal: Infection and Drug Resistance

\begin{abstract}
Herpes zoster (HZ) is an acute vesicular dermatitis with a typical dermatomal distribution, caused by the varicella zoster virus (VZV), often preceded and accompanied by prodromal pain or pruritus. HZ may be related to several complications such as postherpetic neuralgia (PHN). The incidence and severity of the disease increase with aging, due to immunosenescence and in particular to the decline of the specific cell-mediated immunity (CMI). The impact of $\mathrm{HZ}$ in terms of morbidity and short- and long-term complications, the availability of suboptimal treatment options to date, and the high costs for the diagnostic and clinical-therapeutic management of patients have motivated the search for a new preventive approach through the development of a vaccine. The vaccine currently in use with live-attenuated virus (ZVL) has been shown to be effective in reducing the incidence of HZ, its impact, and the onset of PHN, although the efficacy is lower in older subjects and tends to decrease some years after immunization. A new adjuvanted recombinant subunit vaccine (HZ/su), containing the VZV glycoprotein $\mathrm{E}(\mathrm{gE})$ and the AS01B adjuvant system, is now a very promising alternative to ZVL; in several clinical studies, it showed a good safety profile and was able to elicit high immune humoral and cell-mediated responses, both maintained up to 9 years. Furthermore, HZ/su vaccine was effective both in preventing $\mathrm{HZ}$ and in reducing the onset of PHN and other complications. $\mathrm{HZ} /$ su has been recommended and preferred over ZVL by the Advisory Committee on Immunization Practices (ACIP) for the prevention of $\mathrm{HZ}$ and its complications in immunocompetent adults aged $\geq 50$ years, even if already vaccinated with ZVL, through a two-dose schedule. HZ/ su has been approved in Canada, USA, Europe, and Japan and is currently being approved in Australia. The aim of this review was to describe the epidemiological data, HZ and PHN risks and their impact on the social life and common life of infected people, and ZVL and HZ/su vaccine development including various clinical trials and efficacy, safety, and tolerability profiles. Keywords: herpes zoster, adjuvanted recombinant herpes zoster subunit vaccine, live-attenuated herpes zoster vaccine
\end{abstract}

\section{Background}

Varicella zoster virus (VZV) is an $\alpha$-herpes virus, with a double-stranded DNA genome, that exclusively infects humans. The primary infection causes chickenpox (varicella), during which the virus becomes latent in the ganglia of the nervous system. The mechanism by which cells become latently infected is unclear; it has been hypothesized for a long time, observing the similar interest of the cutaneous areas involved in varicella and herpes zoster (HZ), that the virus enters from the epidermal nerve endings and undergoes a retrograde axonal migration; however, it seems more plausible that the cause of VZV latentization is the varicella-associated viremia that would explain the
Correspondence: Giovanni Gabutti Department of Medical Sciences, University of Ferrara, Via Fossato di Mortara 64/b, 44I2I Ferrara, Italy

Tel +390532455568

Fax +390532 205066

Email giovanni.gabutti@unife.it 
involvement of neurons that do not have cutaneous projections such as enteric ones. ${ }^{1}$

During latency, the subclinical viral activity may induce a VZV-specific cell-mediated immunity (CMI) (endogenous booster) that prevents the clinical reactivation of the virus. ${ }^{2}$

Due to the decline in CMI, typical of the elderly, or as a result of immunocompromising diseases/conditions, the virus can reactivate causing $\mathrm{HZ}$ (or shingles). ${ }^{1}$

$\mathrm{HZ}$ is a vesicular rash with typical dermatomal distribution accompanied by pain; it is often preceded by prodromal pain or pruritus. The risk of developing HZ during the life course is estimated to be $\sim 20-30 \% .{ }^{2}$ Incidence and severity of the disease increase with aging, and it is estimated that $\sim 50 \%$ of subjects aged $\geq 85$ years could have an episode of $\mathrm{HZ}{ }^{3}$

From an epidemiological point of view, the incidence rate of $\mathrm{HZ}$ ranges between 3 and 5/1,000 person-years (py) in Europe, Asia and North America; this rate increases considerably in the elderly (6-8/1,000 py in people aged $\geq 60$ years, $8-12 / 1,000$ py in people aged $\geq 80$ years). ${ }^{4}$

A review on the incidence of $\mathrm{HZ}$ in Europe estimates that there are $1.7 \pm 0.1$ million new cases/year. Incidence rates vary according to age (from $1 / 1,000$ in children aged $<10$ years up to $7-8 / 1,000$ in subjects aged $>50$ years) and reach values of $10 / 1,000$ in subjects aged $\geq 80$ years. $^{5}$

$\mathrm{HZ}$ is related to several complications; the most relevant one is postherpetic neuralgia (PHN), referred to as a pain persisting at least 90 days after the herpetic rash onset. ${ }^{6}$ The risk of developing PHN is estimated between 5 and $30 \%$ of HZ cases, and this variability is due to different study designs and different definitions used. ${ }^{4}$ The risk factors for PHN are aging as well as the severity of the prodromes, pain, and rash of the acute phase. ${ }^{6}$

Pain, present in all phases of the disease, is certainly the symptom with the greatest impact on the quality of life and on the activity of daily living (ADL). $\mathrm{HZ}$ and $\mathrm{PHN}$ have negative effects on the physical, mental, social, and functional health of affected individuals, consequently also influencing the social and family spheres of patients. ${ }^{7}$ A survey conducted in six European countries identified, of the 44,545 individuals aged $>50$ years, 1,005 people who had an episode of $\mathrm{HZ} ; 13 \%$ of these subjects developed PHN. The association between HZ and pain and even more markedly between PHN and pain was confirmed; besides, a strong impact on the quality of life of patients and of their friends and family was also highlighted. ${ }^{8}$

$\mathrm{HZ}$ ophtalmicus (HZO) is another relevant complication; it occurs when the ophthalmic branch of the trigeminal nerve is affected. An incidence of 30.9/100,000 py is reported, while the risk of developing HZO varies between 10.1 and $14.9 \%$ of $\mathrm{HZ}$ cases. $\mathrm{HZO}$ can occur without or with the involvement of the eye, and there is a risk of between 30 and $78 \%$ of ocular complications (keratitis, uveitis, and conjunctivitis) that can lead to a loss of vision. ${ }^{4}$

$\mathrm{HZ}$ seems to increase the risk for other diseases; for example, two meta-analyses show an increased risk of stroke after $\mathrm{HZ}$ infection. This risk seems to be higher within the first month of infection. An increased risk of cerebral ischemic events after $\mathrm{HZO}$ compared to $\mathrm{HZ}$ episodes in the absence of such complication has been reported. ${ }^{9,10}$

Zhang et al also showed an albeit minor but still significant association between $\mathrm{HZ}$ and myocardial infarction. ${ }^{9}$

Regarding the HZ impact in terms of costs and use of health resources, the hospitalization rate is estimated to range between 2 and 25/100,000 py, depending on the admission criterion used; the hospitalization rate increases with aging, in particular after the age of 50 years. ${ }^{4}$ An Italian study, performed in the period 2001-2013, showed that 16.1, 27.6, and $56.3 \%$ of hospital admissions involved $<49-, 50-69-$, and $>70$-year-old subjects, respectively; the global rate was equal to 12.1 hospitalizations/100,000 inhabitants/year. ${ }^{11}$

$\mathrm{HZ}$ also has a strong impact in terms of health costs. Direct costs due to treatment, hospitalizations, medical and diagnostic examinations, and other health resources have the greatest economic weight. The advanced age, the presence of comorbidity or immunosuppression, the duration of hospitalization, the use of drugs, and the severity of symptoms involve high direct costs. The indirect costs (absent from work and loss of productivity) concern subjects of working age and are therefore associated with the age of onset of the disease. ${ }^{12}$

The treatment of HZ involves the use of antiviral drugs to be started possibly within the first 3 days after the onset of the disease. This therapy improves pain due to $\mathrm{HZ}$ but has usually no effect on reducing the risk of PHN. Treatment of this complication is difficult and is based on symptomatic drugs (lidocaine and capsaicin for local use, gabapentin and pregabalin, and tricyclic antidepressants). Combination therapy or opioid use can be performed; however, it seems that PHN-related pain is alleviated only in half of the cases. ${ }^{1}$

As a whole, the impact of $\mathrm{HZ}$ in terms of morbidity and short- and long-term complications, the availability of suboptimal treatment options, and the high costs for the diagnostic and clinical therapeutic management of patients have motivated the search for a new preventive approach through the development of a vaccine. ${ }^{13}$ 


\section{Live-attenuated $\mathrm{HZ}$ vaccine (ZVL)}

Due to immunosenescence, there is a progressive decline in the VZV-specific CMI, associated with a higher incidence and severity of $\mathrm{HZ}$ and PHN in the elderly; otherwise, the levels of VZV-specific antibodies remain relatively constant with advancing age. An episode of $\mathrm{HZ}$ causes a reactivation (booster effect) of the long-lasting $\mathrm{CMI} ;{ }^{14}$ therefore, the risk of recurrence of $\mathrm{HZ}$ is quite low in immunocompetent subjects. ${ }^{15}$ In light of this evidence, it has been hypothesized that, by stimulating the specific cell-mediated response, the frequency and severity of $\mathrm{HZ}$ and its complications could be reduced. This target has been pursued developing a liveattenuated vaccine with high antigen content.

The live-attenuated $\mathrm{HZ}$ vaccine, approved and currently in use (ZVL; Zostavax; Merck Sharpe \& Dohme Corp, USA), consists of the same strain used in the pediatric varicella vaccine (Oka/Merck strain), with a high antigenic content; the latter is at least 14 times higher $(19,400$ plaque-forming units [PFU]) than the pediatric VZV live-attenuated vaccine.

ZVL must be stored and transported refrigerated $\left(2-8^{\circ} \mathrm{C}\right)$ and administered subcutaneously or intramuscularly in a single dose $(0.65 \mathrm{~mL}){ }^{16}$

Efficacy, safety, and effectiveness of ZVL have been extensively studied.

The Shingles Prevention Study (SPS), a double-blind multicenter randomized controlled clinical trial, enrolled $>38,000$ subjects aged $\geq 60$ years, with a history of chickenpox or of living for $>30$ years in an endemic area. The subjects enrolled received a dose of vaccine or placebo and were followed up $\sim 3$ years to evaluate the efficacy against the burden of illness (BOI, which includes incidence, severity, and duration of acute and chronic HZ-related pain during a 6-month follow-up), PHN, and the incidence of HZ.

ZVL showed an efficacy of $61.1,66.5$, and $51.3 \%$ in reducing the $\mathrm{BOI}$ and the incidence of $\mathrm{PHN}$ and of $\mathrm{HZ}$, respectively, with the excellent safety and tolerability profiles. A lower level of efficacy was found in preventing the occurrence of $\mathrm{HZ}$ in older subjects (63.9\% in the 60-69 years age group vs $37.6 \%$ in the $\geq 70$ years age group), while no differences were found related to the gender. The average duration of pain, discomfort, and severity of the disease were lower in the vaccinated subjects than in the placebo group. The effect of the ZVL in reducing the severity of the disease was greater in older subjects, although the reduction in incidence was, on the contrary, greater in younger age groups. ${ }^{14}$

Another double-blind, randomized, controlled trial that integrated the SPS results was the Zoster Efficacy and Safety Trial (ZEST), in which the same criteria of inclusion and exclusion of the SPS were used enrolling a population of $\sim 22,500$ healthy subjects aged between 50 and 59 years. The enrolled subjects received a dose of vaccine or placebo and were followed up for at least 1 year; the efficacy against HZ was $69.8 \%$. Therefore, the results of both studies showed a similar level of efficacy in the 50-59- and 60-69-year-old subjects; in these age groups, the efficacy was higher than in $\geq 70$-year-old subjects (37.6\%), probably due to a better immune response in younger subjects (Figure 1). ${ }^{17}$ The vaccine was also effective in improving functional status and reducing the interference of $\mathrm{HZ}$ on daily activities and quality of life. ${ }^{18}$

The duration of efficacy was further investigated in the short-term persistence substudy (STPS) ${ }^{19}$ and the long-term persistence study (LTPS $)^{20}$ evaluating a subset of subjects enrolled in the SPS. Recently, the summary of the characteristics of the product (SCP) has been updated with some data on the duration of protection. In detail, the vaccine efficacy against HZ 3 years after vaccination was $60,55,50$, and $48 \%$ in $50-59-, 60-69-, 70-79-$, and $>80$-year-old subjects, respectively. The efficacy against $\mathrm{HZ} 5$ years after vaccination was 49,46 , and $44 \%$ in 60-69-, 70-79-, and $>80$-year-old subjects, respectively.

The efficacy against PHN 3 years after immunization was $98,74,73$, and $63 \%$ in 50-59-, 60-69-, 70-79-, and $>80$-year-old subjects, respectively. The efficacy for this end-point 5 years after immunization was 72,69 , and $61 \%$ in the $60-69,70-79$, and $>80$ years age groups, respectively. ${ }^{21}$

During the last years, it has been verified that the results of efficacy as well as those of effectiveness studies are consistent. In particular, the effectiveness data are consistent with each other, although they come from studies carried out with different designs and different characteristics of the enrolled populations (Figure 1). ${ }^{22-30}$

All studies showed the good safety and tolerability profiles of ZVL.

ZVL is indicated in subjects aged $>50$ years; it can be administered to patients with a history of $\mathrm{HZ}$ and also to subjects whose clinical history is unknown. Currently, there are no indications for the administration of ZVL to those who have already received the varicella vaccine..$^{31} \mathrm{ZVL}$ is contraindicated in subjects with a history of hypersensitivity to any component of the vaccine, suffering from primitive or acquired immunodeficiency, on immunosuppressive therapy (including high doses of corticosteroids), with untreated active tuberculosis and pregnancy. ${ }^{13} \mathrm{ZVL}$ should be administered 14-30 days before the start of any immunosuppressive treatment. ${ }^{31,32}$ 


\section{A}

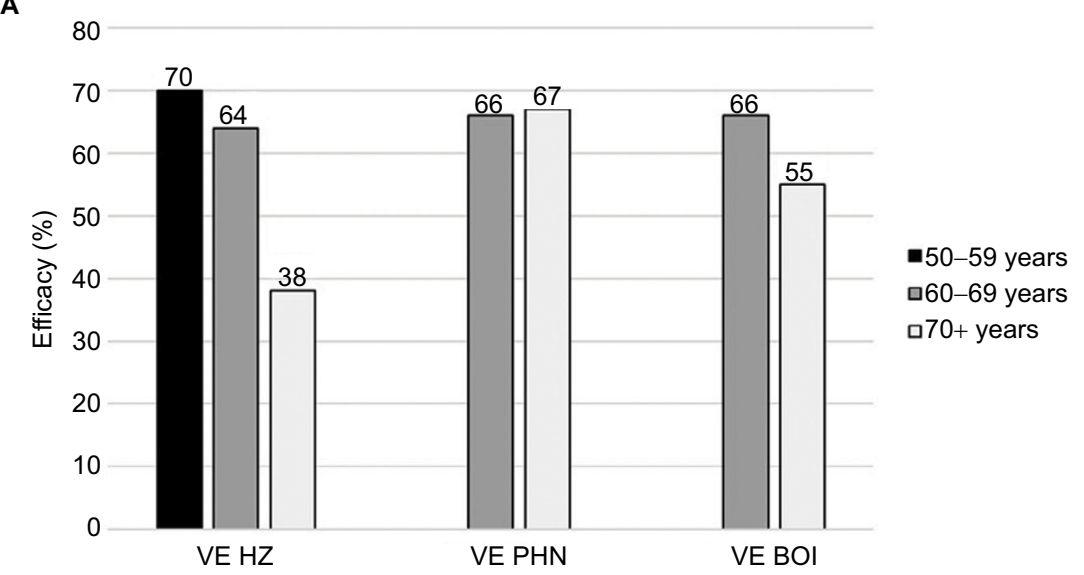

B

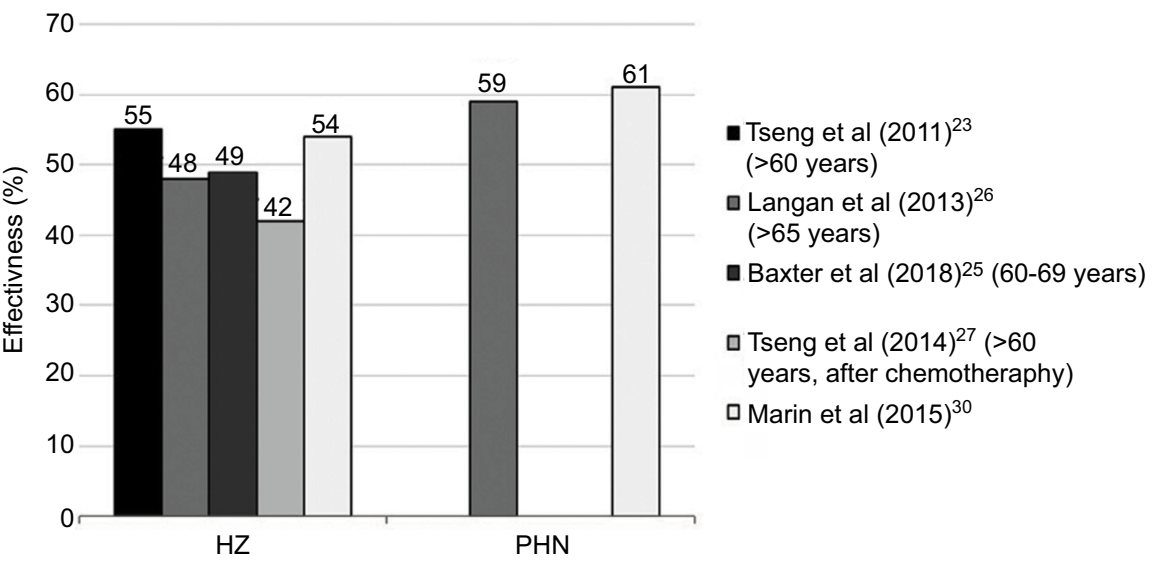

Figure I Efficacy ${ }^{14,17}(\mathbf{A})$ and effectiveness ${ }^{23,25-27,30}(\mathbf{B})$ of the live-attenuated $\mathrm{HZ}$ vaccine (ZVL).

Abbreviations: $\mathrm{BO}$, burden of illness; $\mathrm{HZ}$, herpes zoster; PHN, postherpetic neuralgia; $\mathrm{VE}$, vaccine efficacy.

ZVL can be co-administered with the flu vaccine, provided it is injected into different anatomical sites, but it would not be co-administered with the 23 -valent pneumococcal vaccine (PPSV23) because, as shown in a randomized clinical study, it causes a reduced humoral immune response to ZVL compared with administration of the vaccines 4 weeks apart. $^{13,33}$

However, as protection against $\mathrm{HZ}$ is mainly maintained by VZV-specific T CMI, a cohort study did not find any difference in the protection against $\mathrm{HZ}$ when ZVL was coadministered with PPSV23 in respect to a 4 weeks separate administration. $^{34}$

These observations were recently confirmed by a retrospective cohort study, which showed that there is no lack of protection against $\mathrm{HZ}$ following the co-administration of the two vaccines compared to their separate administration. ${ }^{35}$

Several countries in the world have already included or foresee to include $\mathrm{HZ}$ vaccination in their immunization calendar. Although the strategies of use of the ZVL vaccine worldwide are heterogeneous, there is a clear convergence on the definition of the vaccination targets based on age group cohorts with greater burden of disease and the presence of risk factors starting from the age of 50 years. Where immunization has been adopted and implemented, the results are very interesting. For example, the UK data for the first 3 years after the introduction of ZVL showed a decrease of $\sim 17,000$ and 3,300 cases of $\mathrm{HZ}$ and $\mathrm{PHN}$, respectively, in a population of 5.5 million eligible subjects for the vaccination program. The first assessment of vaccine efficacy in UK demonstrated a good impact on both $\mathrm{HZ}$ and $\mathrm{PHN} .^{36,37}$

\section{Adjuvanted recombinant $\mathrm{HZ}$ subunit vaccine}

An alternative option to the ZVL vaccine is the adjuvanted recombinant $\mathrm{HZ}$ subunit vaccine $(\mathrm{HZ} / \mathrm{su}$ ) containing the glycoprotein $\mathrm{E}$ (gE) of the VZV and the adjuvant system AS01B. 
The $\mathrm{gE}$ has been selected as a vaccinal antigen because it is the most expressed viral protein, is essential for the intercellular viral replication and diffusion, and is one of the targets of the VZV-specific immune response..$^{38} \mathrm{gE}$ is able to stimulate the humoral immune response, through the production of neutralizing antibodies, and also the cell-mediated response, through the activation of $\mathrm{CD}^{+}{ }^{+} \mathrm{T}$ cells. ${ }^{39}$ The AS01B is a liposomal adjuvant that contains $50 \mu \mathrm{g}$ of 3-O-desacil-4"monophosphoryl lipid A and $50 \mu \mathrm{g}$ of the saponin Quillaja saponaria, fraction 21 (QS21), which stimulates the activation of the innate immune response, the production of cytokines, and the recruitment of antigen-presenting cells. ${ }^{39,40}$ The monophosphoryl lipid A, in synergy with QS21, stimulates the production of $\gamma$-interferon and, therefore, enhances the immune response, acting as a toll-like receptor four agonist. ${ }^{41}$ As demonstrated in preclinical studies, ${ }^{42}$ the AS01B adjuvant also enhances the specific antibody and cell-mediated response to the recombinant protein. ${ }^{38}$ In animal models, $\mathrm{gE}$ stimulated a higher activation of $\mathrm{CD}^{+} \mathrm{T}$ lymphocytes when associated with the adjuvant AS01B compared to when administered in the absence or with another adjuvant. ${ }^{42}$

In 2012, a Phase I/II study was conducted in Belgium by Leroux-Roels et al with the aim of investigating the safety and immunogenicity profiles of the subunit vaccine compared to the live-attenuated vaccine (VZV pediatric vaccine). The study showed that $\mathrm{HZ} /$ su induces a greater immune response, both cell mediated and humoral, which remains higher than the one obtained using VZV pediatric vaccine up to 42 months both in young adults (18-30 years) and in older subjects (50-70 years).

This trial also showed an acceptable safety profile of the $\mathrm{HZ} / \mathrm{su}$ vaccine, despite a greater frequency of nonsevere adverse reactions (such as asthenia, myalgia, headache, and pain at the injection site) compared to the VZV pediatric vaccine. ${ }^{43}$

Even the Phase I study by Lal et $\mathrm{al}^{44}$ with the administration of two doses of $\mathrm{HZ}$ vaccine (2-month interval) on healthy volunteers in Japan showed an acceptable safety profile.

In 2013, Chlibek et al conducted a multicentric and international Phase II trial with the aim of comparing the efficacy and safety of different adjuvanted subunit vaccines compared to the same vaccine without adjuvant. The study involved $\geq 50$-year-old adults randomly divided into four groups who were given the $\mathrm{gE}$ adjuvanted subunit vaccine containing either the liposomal complex AS01B (HZ/su) or the AS01E (gE/AS01E; AS01E contains $500 \mu \mathrm{g}$ of dioleoyl phosphatidylcholine, $125 \mu \mathrm{g}$ of cholesterol, $25 \mu \mathrm{g}$ of Monophosphoryl Lipid A, and $25 \mu \mathrm{g}$ of QS21), the nonadjuvanted subunit vaccine (gE), and placebo, respectively. The results showed that the immune response is significantly higher with the two adjuvanted vaccines in comparison to the nonadjuvanted vaccine and that the $\mathrm{HZ} / \mathrm{su}$ vaccine provides a better response than the adjuvanted $\mathrm{gE} / \mathrm{AS} 01 \mathrm{E}$ vaccine.

In contrast, as far as tolerability is concerned and adjuvanted vaccines have shown a greater occurrence of adverse reactions, all of which transient and of mild-to-moderate degree, such as pain at the injection site among local reactions and asthenia among the general ones. ${ }^{45}$

In 2014, Chlibek et al conducted another Phase II study based on the experience of the previous year. In this study, the investigators wanted to look for the safety and immunogenicity profiles between different formulations and schedules of the gE subunit vaccine adjuvanted with AS01B. The study design enrolled 714 subjects aged $\geq 60$ years randomly divided into five groups. Different formulations/schedules were used, as follows: one single-dose of gE/AS01B vaccine with a $100 \mu \mathrm{g}$ of $\mathrm{gE}$, two doses of gE/AS01B (2 months apart) with 25,50 , or $100 \mu \mathrm{g}$ of $\mathrm{gE}$, and two doses of nonadjuvanted $\mathrm{gE}$ vaccine ( 2 months apart) with $100 \mu \mathrm{g}$ of $\mathrm{gE} .^{46}$ The results, analyzed at 1, 2, 3, 12, 24, and 36 months after the first administration, showed that the two-dose schedule of adjuvanted vaccine leads to a gE-specific clonal expansion of $\mathrm{CD}^{+} \mathrm{T}$ lymphocytes. This expansion was registered with all formulations analyzed and was three times higher than the one obtained with the monoadministration of adjuvanted vaccine or the two-dose administration of the nonadjuvanted vaccine. Furthermore, immune response remained high over time. The different formulations showed that the humoral response induced with the administration of two doses of 50 or $100 \mu \mathrm{g}$ of $\mathrm{gE} / \mathrm{AS} 01 \mathrm{~B}$ is similar and superior to that obtained in the other groups; the nonadjuvanted $\mathrm{gE}$ vaccine was found to be able to elicit the humoral response but not the cell-mediated one. Besides, this study confirms the greater reactogenicity of the adjuvanted vaccine compared to the nonadjuvanted vaccine; however, reactogenicity does not change significantly taking into account the different formulations.

In 2016, Chlibek et $\mathrm{al}^{47}$ published a further Phase II trial with the aim of assessing the vaccine-induced immune response in the cohort of immunized subjects enrolled in the previous study performed in 2014. In this study, the follow-up involved only those belonging to the group that received the $\mathrm{HZ} /$ su vaccine (gE/AS01B adjuvanted vaccine with the $50 \mu \mathrm{g}$ formulation) and evaluated the duration of cell-mediated and humoral immune responses at 48, 60, and 72 months after the first dose. The results showed that after 72 months both 
cell-mediated and humoral responses tend to decrease by 20-25\% compared to the 36 th month, remaining however satisfactory and significantly higher than the prevaccination level. In detail, the $\mathrm{HZ} / \mathrm{su}$ vaccine provides a 3.8 times higher cell-mediated response and a 7.3 times higher humoral response than the preimmunization status.

In 2018, Schwarz et al made a further extension of the previous study evaluating humoral and cell-mediated immune responses 9 years after the first dose. The results of the follow-up have confirmed that, even in this case, the immunity persists significantly above the preimmunization level regardless of the age of the analyzed subjects. In particular, the study estimated that this immunity will remain high up to at least 15 years after vaccination. ${ }^{48}$

A study that provided relevant information in defining the optimal schedule in terms of time interval between the two doses of $\mathrm{HZ} / \mathrm{su}$ vaccine was conducted by Lal et al in 2018. In this study, the average geometric titers (GMTs) were analyzed 1 month after the second dose of vaccine given accordingly to a $0-2,0-6$, and $0-12$ months' schedule. The study demonstrated an excellent immune response in the 0-6 months' schedule group, comparable to that obtained in the 0-2 months' schedule group. Besides, GMTs were lower using the 0-12 months' schedule; this suggests that the second dose of $\mathrm{HZ} /$ su should be optimally (ie, without loss of efficacy) administered in a time window between 2 and 6 months after the first dose. ${ }^{49}$

Another point studied in the approval trials of the $\mathrm{HZ} / \mathrm{su}$ vaccine has been the evaluation of the efficacy of the vaccination on anamnestically positive subjects for $\mathrm{HZ}$ and on subjects already vaccinated with ZVL.

In 2017, Godeaux et al analyzed, in a Phase III trial, the immunogenicity of the vaccine in a cohort of $\geq 50$-year-old subjects with previous and documented history of $\mathrm{HZ}$ stratified by age group. The $\mathrm{HZ} / \mathrm{su}$ vaccine turned out to elicit a high immune response and to have a good safety profile. ${ }^{50}$

In the same year, following the documented decrease in the immune response 3-7 years after vaccination with ZVL, Grupping et $\mathrm{al}^{51}$ evaluated the possibility of administering the $\mathrm{HZ} /$ su vaccine even to ZVL recipients. This open-label case-control study enrolled subjects $>5$ years previously vaccinated with ZVL and subjects never vaccinated (ZVL naive). The results demonstrate that $\mathrm{HZ} / \mathrm{su}$ induces a strong activation of both humoral and cell-mediated immune responses regardless of the previous vaccination status and that the hypothesis of revaccinating subjects already vaccinated with ZVL may be considered.
In 2017, Vink et al published a Phase III study conducted on 60 Japanese subjects aged $\geq 50$ years evaluating the safety and immunogenicity of $\mathrm{HZ} / \mathrm{su}$ vaccine when administered subcutaneously vs intramuscularly. The results of the study showed a comparable immune response between subcutanous administration and intramuscular administration; however, a greater reactogenicity was registered using the subcutaneous route of admnistration. ${ }^{52}$

The development of a nonlive-inactivated vaccine for the prevention of $\mathrm{HZ}$ represents an important tool to be available in subjects with impaired immune status. As a matter of fact, these patients, notwithstanding their greater risk of contracting the disease and developing severe complications, until today could not undergo vaccination due to the risk possibly related to the administration of the live-attenuated vaccine (ZVL). ${ }^{53}$ In this regard, studies conducted by Berkowitz et $\mathrm{al}^{54}$ and by Stadtmauer et $\mathrm{al}^{55}$ on HIV-infected subjects and hematopoietic cell transplant recipients, respectively, are very relevant.

The first study, conducted on $\geq 18$-year-old HIV+ subjects and stratified according to the $\mathrm{CD}^{+} \mathrm{T}$-cell count in three groups ( $\geq 200,50-199$, and $\geq 500$ cells $/ \mathrm{mm}^{3}$ ), evaluated the efficacy and safety profiles of the HZ/su compared to placebo group. The results showed that both VZV-specific humoral and cell-mediated immunities reached high values, with a peak after the second dose, persisting for at least 18 months. In addition, from the clinical point of view, the $\mathrm{HZ} / \mathrm{su}$ was found to be safe and well tolerated in these patients without any effect on the HIV viral load. ${ }^{54}$

The second one was a case-control study conducted on 121 patients with multiple myeloma, non-Hodgkin lymphoma (B cell or T cell), Hodgkin lymphoma, or acute myeloid leukemia who had undergone autologous hematopoietic stem-cell transplant 50-70 days earlier. These patients were randomly divided into four groups who, according to the 0-1-3 months' schedule, received three doses of $\mathrm{HZ} / \mathrm{su}$, three doses of gE/AS01E, one dose of placebo, and two doses of $\mathrm{HZ} /$ su or three doses of placebo, respectively. A high immune response, both humoral and cell mediated, was achieved with the three-dose schedule of the adjuvanted vaccine independently of the adjuvant used. In addition, the safety profile was judged adequate, since most reactions were classified as mild-moderate. ${ }^{55}$

A recent clinical trial, not yet published, was conducted in autologous hematopoietic cell transplant recipients aged 18 years and older to evaluate the efficacy of $\mathrm{HZ} / \mathrm{su}$ in the prevention of $\mathrm{HZ}$. 
The results show high efficacy in the prevention of $\mathrm{HZ}$ and PHN with a very significant reduction in the duration of "worst" HZ-associated pain calculated in days vs placebo. ${ }^{56}$

In 2017, Schwarz et al investigated the co-administration of $\mathrm{HZ} / \mathrm{su}$ and quadrivalent seasonal inactivated influenza vaccine, in order to evaluate the immunogenicity and safety vs the single administration of the vaccines in different times.

The results of the study show no interference in the immune responses to either vaccine when the vaccines were co-administered, and no safety concerns were identified. ${ }^{57}$

In addition to numerous and relevant studies aimed at investigating the immunogenicity, safety, and tolerability of the $\mathrm{HZ} /$ su vaccine, two approval pivotal trials published by Lal et al $(\mathrm{ZOE}-50)^{38}$ and by Cunningham et al (ZOE- 70$)^{40}$ in 2015 and 2016, respectively, have been undertaken in order to evaluate its impact on the reduction in the disease and its complications.

The ZOE-50 is a multicenter randomized study conducted in 18 countries enrolling 15,411 subjects aged $\geq 50$ years divided in two groups receiving two doses of HZ/su or two doses of placebo 2 months apart, respectively. During an average follow-up time of 3.2 years, 210 and six cases of $\mathrm{HZ}$ were registered in the placebo group and in the HZ/ su group, respectively. Thus, the efficacy of HZ/su against $\mathrm{HZ}$ was $97.2 \%$. In addition, the efficacy in subjects aged $\geq 70$ years was found to be comparable to that registered in younger subjects.
As in previous studies, however, the HZ/su group reported more frequently, than the placebo group, the occurrence of solicited local and systemic reactions in the first 7 days, most of them of mild-to-moderate intensity and transient. ${ }^{38}$ The ZOE-70 study continues the experience of the ZOE-50 study with the enrollment of 13,900 older (aged $\geq 70$ years) subjects randomized to receive two doses of $\mathrm{HZ} / \mathrm{su}$ or placebo; in both groups, a 2-month interval between doses was used. As in the study conducted on younger patients, also in this case, there was a clear difference in the occurrence of $\mathrm{HZ}$ cases (23 and 223 in the vaccinated group and in the placebo group, respectively) with an estimated efficacy of $89.8 \%$ in the reduction in the appearance of the disease at a mean follow-up period of 3.7 years. ${ }^{40}$

In addition to the abovementioned high efficacy of the $\mathrm{HZ} / \mathrm{su}$, it is important to highlight the impressive reduction in the risk of developing complications $\mathrm{HZ}$ correlated, such as $\mathrm{PHN}$, in vaccinated subjects compared with the placebo group (Figure 2). ${ }^{58}$

A predefined pooled analysis considering all together $(16,596)$ subjects aged $\geq 70$ years enrolled in both ZOE-50 and ZOE-70 studies estimated the efficacy of the vaccine against HZ (91.3\%) and PHN (88.8\%) in the older population. In the same analysis, no relevant waning of $\mathrm{HZ} / \mathrm{su}$ efficacy against $\mathrm{HZ}$ was found from year $1(97.6 \%)$ to year $4(87.9 \%)$. Regarding the safety profile of $\mathrm{HZ} /$ su vaccine, compared to placebo, this study also confirmed a greater

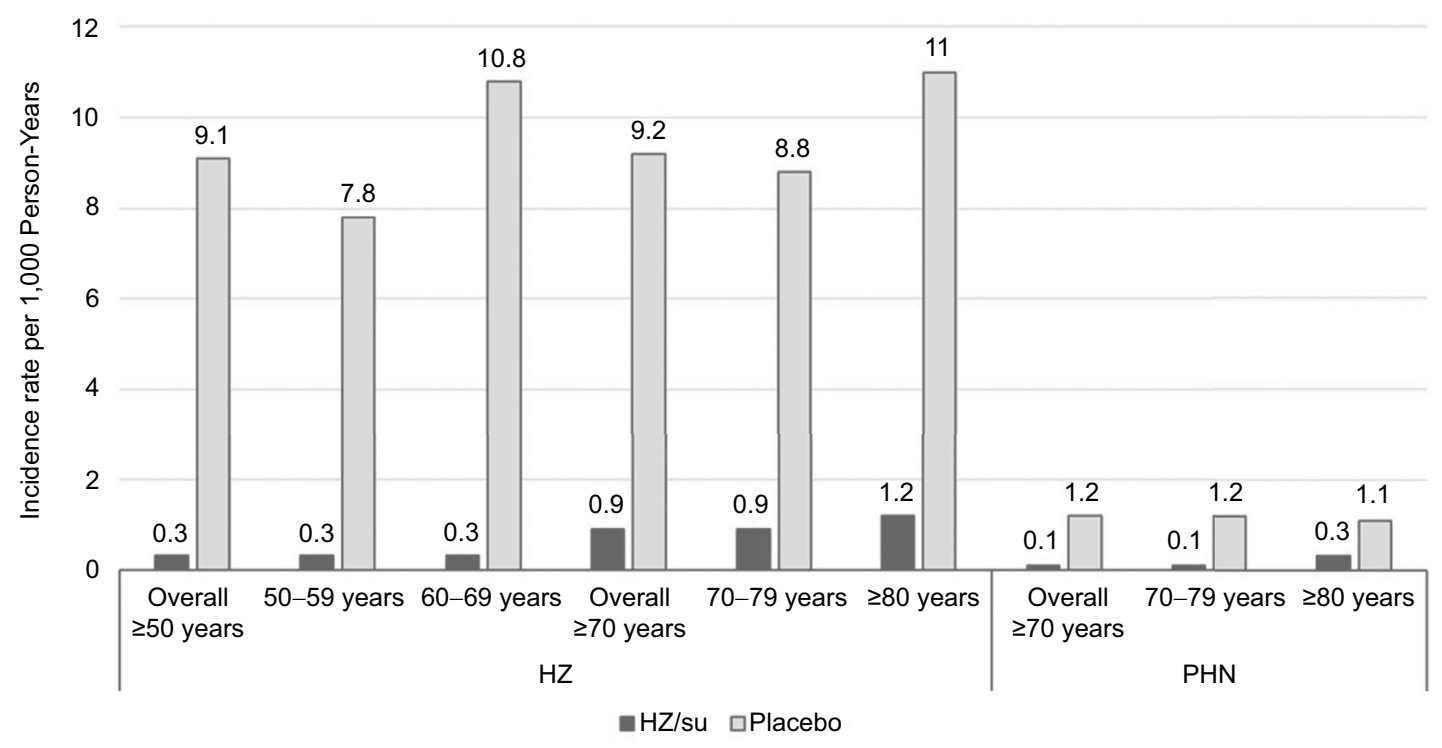

Figure 2 Incidence rate of $\mathrm{HZ}$ and $\mathrm{PHN}$ per I,000 Person-Years in $\mathrm{HZ} /$ su group and in placebo group.

Note: Data from Food and Drugs Administration (FDA). Shingrix Highlights of Prescribing Information. Available from: https://www.fda.gov/downloads/biologicsbloodvaccines/ vaccines/approvedproducts/ucm581605.pdf. Accessed May 14, 2018. ${ }^{58}$

Abbreviations: $\mathrm{HZ}$, herpes zoster; $\mathrm{HZ} /$ su, $\mathrm{HZ}$ subunit vaccine; $\mathrm{PHN}$, postherpetic neuralgia. 
frequency of occurrence of mild or moderate local or systemic adverse reactions in recipients ( 79 vs 30\% in the placebo group). Regarding severe reactions, deaths, and potential immune-mediated diseases, no statistically significant differences were observed between the two groups in both ZOE studies and the compliance of the subjects enrolled in carrying out the whole two-dose vaccination cycle with $\mathrm{HZ} /$ su was very high. ${ }^{40,59}$

As reported in the technical data sheet of $\mathrm{HZ} / \mathrm{su}$, the effect on the reduction of disease commitment (BOI) was $98.4 \%$ in subjects aged $\geq 50$ years (ZOE-50) and $92.1 \%$ in subjects aged $\geq 70$ years (ZOE-50 and ZOE-70 together). The BOI score includes the incidence of $\mathrm{HZ}$ with the severity and duration of acute pain and chronic pain associated with $\mathrm{HZ}$ over a period of 6 months following the onset of rash. ${ }^{60}$

In 2018, Kovac et al analyzed data from the ZOE-50 and ZOE-70 studies in order to evaluate further information from the follow-up of enrolled patients such as mortality, number of hospitalizations, and HZ complications (excluding PHN already analyzed by the previous study) defined as HZ-related vasculitis, stroke, and disease with neurological and ophthalmic involvements. The results showed that the efficacy of the vaccine in reducing the impact of the aforementioned HZ complications has been 93.7 and $91.6 \%$ in $\geq 50$ - and $\geq 70$-year-old subjects, respectively. Furthermore, during the follow-up, five hospitalizations for $\mathrm{HZ}$ were registered, all belonging to the group receiving placebo; no HZ-related deaths were recorded in both groups. ${ }^{61}$

\section{Pharmaco-economic evaluations}

An absolutely relevant point coming out from the recent literature on the use of the $\mathrm{HZ} / \mathrm{su}$ vaccine is its potential impact on public health and cost-effectiveness.

In this regard, the study of Curran et al, conducted on the basis of the results of efficacy studies in the prevention of $\mathrm{HZ}$ and $\mathrm{PHN}$, estimated the impact of vaccination with $\mathrm{HZ} /$ su on the German population aged $\geq 50$ years, comparing it with the use of ZVL. The results were analyzed dividing the population into three age groups (50-59, 60-69, and $>70$ years) and assuming a $40 \%$ coverage for both vaccines and a $70 \%$ coverage for the second dose of $\mathrm{HZ} / \mathrm{su}$. According to the authors, the HZ/su vaccine would allow, in the three age groups and from the time of vaccination to the end of life, to avoid 725,233, 533,162, and 486,794 cases vs $198,477,196,000$, and 104,640 cases in respect to the ZVL vaccine. The number needed to vaccinate (NNV) to prevent a case of $\mathrm{HZ}$ would be between 8 and 11 for $\mathrm{HZ} / \mathrm{su}$ vs 20-50 for ZVL, and the NNV to prevent a case of PHN would range between 39 and 53 vs 94 and 198 for HZ/su and ZVL, respectively. ${ }^{62}$

Also in a recent experience, published by Watanabe et al, ${ }^{63}$ the potential impact on the Japanese population of $\mathrm{HZ} / \mathrm{su}$ vaccination compared to ZVL was estimated.

The results of vaccination with $\mathrm{HZ} /$ su could prevent $\sim 3.3$ million HZ cases, 692,000 cases of PHN, and 281,000 cases of other complications, compared with the prevention of 0.8 million $\mathrm{HZ}$ cases, 216,000 PHN cases, and 57,000 other complications with vaccination with ZVL. The NNV in order to prevent one $\mathrm{HZ}$ case would range between 6 and 14 using $\mathrm{HZ} /$ su and between 21 and 138 using ZVL. ${ }^{63}$

Also, from the pharmaco-economic point of view, the HZ/ su vaccine guarantees a significant impact in terms of optimization of public health spending, with a high probability of being cost-effective, as demonstrated by the study by Le et al. ${ }^{64}$ The authors estimated that at a cost of $\$ 280$ ( $\$ 140$ per dose), $\mathrm{HZ} /$ su would be more effective and less expensive than ZVL in all age groups considered ( $\geq 60, \geq 70$, and $\geq 80$ years) while the Incremental Cost-Effectiveness Ratio (ICER) of vaccination with $\mathrm{HZ} /$ su compared to nonvaccination would range between 20,038 and \$30,084 per QALY, according to the age considered.

Always considering the price of $\$ 280$ per vaccination cycle, HZ/su would have $73-91 \%$ probability, depending on the age groups considered, of being cost-effective at $\$ 50,000$ per QALY and 78-93\% probability at \$100,000 per QALY. In comparison, at the same threshold of $\$ 100,000$ per QALY, ZVL vaccine given to 60 -year-old subjects would be costeffective with the probability of $<5 \%{ }^{64}$

\section{$\mathrm{HZ} /$ su: approvals and recommendations}

Based on the excellent and promising results of the clinical trials, the HZ/su vaccine, named now Zoster Vaccine Recombinant, Adjuvanted (RZV, Shingrix; GlaxoSmithKline plc, London, UK), has been approved for the first time worldwide in Canada on October 13, 2017, and a few days later (on October 20, 2017) in the USA by the Food and Drug Administration (FDA).

With the approval of the vaccine, the Advisory Committee on Immunization Practices (ACIP) of the Centers for Disease Control (CDC) has made public its indications on the basis of a review and analysis of literature and epidemiological data started in March 2015.

The recommendations of the ACIP can be summarized in three points:

1. $\mathrm{RZV}$ is recommended for the prevention of $\mathrm{HZ}$ and its complications in immunocompetent adults aged $\geq 50$ years. 
2. $\mathrm{RZV}$ is recommended for the prevention of $\mathrm{HZ}$ and its complications in immunocompetent adults aged $\geq 50$ years previously vaccinated with $Z \mathrm{VL}$.

3. $\mathrm{RZV}$ is preferable to $\mathrm{ZVL}$ for the prevention of $\mathrm{HZ}$ and related complications.

The optimal route of administration is intramuscular, and it is possible to co-administer RZV with other vaccines in another anatomical site in order to improve patient compliance to vaccination; more specifically, RZV can be administered together with the quadrivalent influenza vaccine without any immunogenicity and safety concern. ${ }^{65}$

Besides, RZV can be co-administered with PPSV23 vaccine as showed in a recent study: immunogenicity of both vaccines is not negatively influenced and co-administration was well tolerated and safe. ${ }^{66}$

The ideal schedule must be with two doses with a 2-6 months' interval; it is important to underline that there is no indication to repeat the vaccination cycle if the second dose is administered $>6$ months after the first one, while if the interval is $<4$ weeks, it would be necessary to consider the first dose null.

The vaccine should not be administered in subjects with active HZ, since it has only preventive and nontherapeutic value, and in pregnant women due to the lack of clinical studies. The only contraindication to vaccination is related to a documented history of a severe allergic reaction to one or more components of the vaccine.

ACIP strongly recommends to administer RZV in subjects with a history of previous $\mathrm{HZ}$, to prevent a possible recurrence of disease, and in patients with comorbidities such as chronic renal failure, diabetes mellitus, rheumatoid arthritis, and COPD for which $\mathrm{HZ}$ can have a great and severe impact on their health condition. RZV is not indicated for the prevention of varicella and only for $\mathrm{HZ}$.

Regarding immunocompromised subjects, ACIP currently recommends vaccination only in patients in remission from the immunosuppressive state, in anticipation of a future immunosuppression, or in subjects who take a low dose of immunosuppressive therapy. As in immunosuppressed patients, the $\mathrm{HZ}$ disease has a greater incidence and impact and the evaluation of the efficacy and safety profiles of the vaccine should be based on more data in addition to those previously reported; ${ }^{65}$ in this regard, some clinical trials are currently underway on immunocompromised subjects in renal transplant recipients ${ }^{67}$ and in chemotherapy for solid ${ }^{68}$ and hematological tumors. ${ }^{69}$

RZV has also been approved for marketing in Europe, thanks to the favorable opinion of the European Medicines
Agency (EMA), and in Japan on March, 23, 2018, and at present, it is pending approval in Australia.

Given the very recent approval of RZV, at the moment, there are no Phase IV trials related to commercialization, which, when available, will provide further, large-scale and relevant information on effectiveness and safety of the new vaccine.

\section{Conclusion}

All evidence available in literature underlines the importance of the new adjuvanted recombinant vaccine (RZV) that seems very promising in the fight against $\mathrm{HZ}$ and its complications. RZV has indeed proved very effective in eliciting a strong immune response, regardless of the age of the immunized subject.

The new recombinant vaccine, moreover, could bring important benefits in immunocompromised subjects for which, despite a higher incidence of $\mathrm{HZ}$, vaccination with live-attenuated virus is contraindicated.

It is very important to underline the strong impact that vaccination with RZV can offer in the context of public health in terms of containing the impact that $\mathrm{HZ}$ and its complications have on the social, economic, and welfare levels. The only critical points of this new vaccine could be related to its reactogenicity and to the need of a two-dose schedule. Therefore, a well-balanced communication will be necessary in order to guarantee a high compliance to this new preventive intervention.

\section{Disclosure}

GG has received grants from GlaxoSmithKline Biologicals SA, Sanofi Pasteur MSD, Novartis, Crucell/Janssen, Seqirus, Sanofi Pasteur, Merck Italy, PaxVax, and Pfizer for being consultant or taking part in advisory board, expert meetings, being a speaker or an organizer of congresses/conferences, and acting as investigator in clinical trials. The authors report no other conflicts of interest in this work.

\section{References}

1. Gershon AA, Breuer J, Cohen JI, et al. Varicella zoster virus infection. Nat Rev Dis Primers. 2015;1:15016.

2. Johnson RW, Wasner G, Saddier P, Baron R. Herpes zoster and postherpetic neuralgia: optimizing management in the elderly patient. Drugs Aging. 2008;25(12):991-1006.

3. WHO. Varicella and herpes zoster vaccines: WHO position paper, June 2014. Wkly Epidemiol Rec. 2014;89(25):265-287. English, French.

4. Kawai K, Gebremeskel BG, Acosta CJ. Systematic review of incidence and complications of herpes zoster: towards a global perspective. $B M J$ Open. 2014;4(6):e004833.

5. Pinchinat S, Cebrián-Cuenca AM, Bricout H, Johnson RW. Similar herpes zoster incidence across Europe: results from a systematic literature review. BMC Infect Dis. 2013;13:170. 
6. Johnson RW, Rice AS. Clinical practice. Postherpetic neuralgia. NEngl J Med. 2014;371(16):1526-1533.

7. Johnson RW, Bouhassira D, Kassianos G, Leplège A, Schmader KE, Weinke T. The impact of herpes zoster and post-herpetic neuralgia on quality-of-life. BMC Med. 2010;8:37.

8. Lukas K, Edte A, Bertrand I. The impact of herpes zoster and postherpetic neuralgia on quality of life: patient-reported outcomes in six European countries. Z Gesundh Wiss. 2012;20(4):441-451.

9. Zhang Y, Luo G, Huang Y, Yu Q, Wang L, Li K. Risk of stroke/transient ischemic attack or myocardial infarction with herpes zoster: a systematic review and meta-analysis. J Stroke Cerebrovasc Dis. 2017;26(8):1807-1816.

10. Marra F, Ruckenstein J, Richardson K. A meta-analysis of stroke risk following herpes zoster infection. BMC Infect Dis. 2017;17(1):198.

11. Valente N, Cocchio S, Stefanati A, et al. Temporal trends in herpes zoster-related hospitalizations in Italy, 2001-2013: differences between regions that have or have not implemented varicella vaccination. Aging Clin Exp Res. 2017;29(4):771-779.

12. Panatto D, Bragazzi NL, Rizzitelli E, et al. Evaluation of the economic burden of herpes zoster (HZ) infection. Hum Vaccin Immunother. 2015;11(1):245-262.

13. Gabutti G, Franco E, Bonanni P, et al. Reducing the burden of herpes zoster in Italy. Hum Vaccin Immunother. 2015;11(1):101-107.

14. Oxman MN, Levin MJ, Johnson GR, et al. A vaccine to prevent herpes zoster and postherpetic neuralgia in older adults. $N$ Engl $J$ Med. 2005;352(22):2271-2284.

15. Tseng HF, Chi M, Smith N, Marcy SM, Sy LS, Jacobsen SJ. Herpes zoster vaccine and the incidence of recurrent herpes zoster in an immunocompetent elderly population. J Infect Dis. 2012;206(2):190-196.

16. Stefanati A, Valente N, Lupi S, et al. Herpes zoster vaccination in the elderly subjects: improving awareness and uptake. Patient Intell. 2015;7:5-20.

17. Schmader KE, Levin MJ, Gnann JW, et al. Efficacy, safety, and tolerability of herpes zoster vaccine in persons aged 50-59 years. Clin Infect Dis. 2012;54(7):922-928.

18. Cook SJ, Flaherty DK. Review of the persistence of herpes zoster vaccine efficacy in clinical trials. Clin Ther. 2015;37(11):2388-2397.

19. Schmader KE, Oxman MN, Levin MJ, et al; Shingles Prevention Study Group. Persistence of the efficacy of zoster vaccine in the shingles prevention study and the short-term persistence substudy. Clin Infect Dis. 2012;55(10):1320-1328.

20. Morrison VA, Johnson GR, Schmader KE, et al; Shingles Prevention Study Group. Long-term persistence of zoster vaccine efficacy. Clin Infect Dis. 2015;60(6):900-909.

21. European Medicines Agency (EMA). SPC Zostavax. Available from: http://www.ema.europa.eu/docs/en_GB/document_library/EPAR_-_ Product_Information/human/000674/WC500053462.pdf. Accessed May 10, 2018.

22. Levin MJ, Schmader KE, Pang L, et al. Cellular and humoral responses to a second dose of herpes zoster vaccine administered 10 years after the first dose among older adults. J Infect Dis. 2016;213(1):14-22.

23. Tseng HF, Smith N, Harpaz R, Bialek SR, Sy LS, Jacobsen SJ. Herpes zoster vaccine in older adults and the risk of subsequent herpes zoster disease. JAMA. 2011;305(2):160-166.

24. Zhang J, Delzell E, Xie F, et al. The use, safety, and effectiveness of herpes zoster vaccination in individuals with inflammatory and autoimmune diseases: a longitudinal observational study. Arthritis Res Ther. 2011;13(5):R174.

25. Baxter R, Bartlett DJ, Fireman B, et al. Long-Term Effectiveness of the Live Zoster Vaccine in Preventing Shingles: A Cohort Study. Am J Epidemiol. 2018;187(1):161-169.

26. Langan SM, Smeeth L, Margolis DJ, Thomas SL. Herpes zoster vaccine effectiveness against incident herpes zoster and post-herpetic neuralgia in an older US population: a cohort study. PLoS Med. 2013;10(4):e1001420.

27. Tseng HF, Tartof S, Harpaz R, et al. Vaccination against zoster remains effective in older adults who later undergo chemotherapy. Clin Infect Dis. 2014;59(7):913-919.
28. Tseng HF, Harpaz R, Luo Y, et al. Declining effectiveness of herpes zoster vaccine in adults aged $\geq 60$ years. $J$ Infect Dis. 2016;213(12): $1872-1875$.

29. Izurieta HS, Wernecke M, Kelman J, et al. Effectiveness and duration of protection provided by the live-attenuated herpes zoster vaccine in the Medicare population ages 65 years and older. Clin Infect Dis. 2017;64(6):785-793.

30. Marin M, Yawn BP, Hales CM, et al. Herpes zoster vaccine effectiveness and manifestations of herpes zoster and associated pain by vaccination status. Hum Vaccin Immunother. 2015;11(5):1157-1164.

31. Maggi S, Gabutti G, Franco E, et al. Preventing and managing herpes zoster: key actions to foster healthy aging. Aging Clin Exp Res. 2015;27(1):5-11.

32. Harpaz R, Ortega-Sanchez IR, Seward JF. Advisory committee on immunization practices (ACIP) centers for disease control and prevention (CDC). Prevention of herpes zoster: recommendations of the advisory committee on immunization practices (ACIP). MMWR Recomm Rep. 2008;57(RR-5):1-30.

33. Macintyre CR, Egerton T, Mccaughey M, et al. Concomitant administration of zoster and pneumococcal vaccines in adults $\geq 60$ years old. Hum Vaccin. 2010;6(11):894-902.

34. Tseng HF, Smith N, Sy LS, Jacobsen SJ. Evaluation of the incidence of herpes zoster after concomitant administration of zoster vaccine and polysaccharide pneumococcal vaccine. Vaccine. 2011;29(20):3628-3632.

35. Bruxvoort K, Sy LS, Luo Y, Tseng HF. Real world evidence for regulatory decisions: concomitant administration of zoster vaccine live and pneumococcal polysaccharide vaccine. Am J Epidemiol. Epub 2018 Apr 11.

36. Amirthalingam G, Andrews N, Keel P, et al. Evaluation of the effect of the herpes zoster vaccination programme 3 years after its introduction in England: a population-based study. Lancet Public Health. 2018;3(2):e82-e90.

37. Walker JL, Andrews NJ, Amirthalingam G, Forbes H, Langan SM, Thomas SL. Effectiveness of herpes zoster vaccination in an older United Kingdom population. Vaccine. 2018;36(17):2371-2377.

38. Lal H, Cunningham AL, Godeaux O, et al. Efficacy of an adjuvanted herpes zoster subunit vaccine in older adults. $N$ Engl $\mathrm{J}$ Med. 2015;372(22):2087-2096

39. Cunningham AL, Heineman TC, Lal H, et al; ZOE-50/70 Study Group. Immune responses to a recombinant glycoprotein $\mathrm{E}$ herpes zoster vaccine in adults aged $\geq 50$ Years. $J$ Infect Dis. 2018;217(11):1750-1760.

40. Cunningham AL, Lal H, Kovac M, et al. Efficacy of the herpes zoster subunit vaccine in adults 70 years of age or older. $N$ Engl J Med. 2016;375(11):1019-1032.

41. Coccia $M$, Collignon $C$, Hervé $C$, et al. Cellular and molecular synergy in AS01-adjuvanted vaccines results in an early IFN $\gamma$ response promoting vaccine immunogenicity. NPJ Vaccines. 2017;2:25.

42. Dendouga N, Fochesato M, Lockman L, Mossman S, Giannini SL. Cell-mediated immune responses to a varicella-zoster virus glycoprotein E vaccine using both a TLR agonist and QS21 in mice. Vaccine. 2012;30(20):3126-3135.

43. Leroux-Roels I, Leroux-Roels G, Clement F, et al. A phase $1 / 2$ clinical trial evaluating safety and immunogenicity of a varicella zoster glycoprotein e subunit vaccine candidate in young and older adults. $J$ Infect Dis. 2012;206(8):1280-1290.

44. Lal H, Zahaf T, Heineman TC. Safety and immunogenicity of an AS01adjuvanted varicella zoster virus subunit candidate vaccine $(\mathrm{HZ} / \mathrm{su})$ : a phase-I, open-label study in Japanese adults. Hum Vaccin Immunother. 2013;9(7):1425-1429.

45. Chlibek R, Bayas JM, Collins H, et al. Safety and immunogenicity of an AS01-adjuvanted varicella-zoster virus subunit candidate vaccine against herpes zoster in adults $>=50$ years of age. $J$ Infect Dis. 2013;208(12):1953-1961

46. Chlibek R, Smetana J, Pauksens K, et al. Safety and immunogenicity of three different formulations of an adjuvanted varicella-zoster virus subunit candidate vaccine in older adults: a phase II, randomized, controlled study. Vaccine. 2014;32(15):1745-1753. 
47. Chlibek R, Pauksens K, Rombo L, et al. Long-term immunogenicity and safety of an investigational herpes zoster subunit vaccine in older adults. Vaccine. 2016;34(6):863-868.

48. Schwarz TF, Volpe S, Catteau G, et al. Persistence of immune response to an adjuvanted varicella-zoster virus subunit vaccine for up to year nine in older adults. Hum Vaccin Immunother. 2018;14(6):1370-1377.

49. Lal H, Poder A, Campora L, et al. Immunogenicity, reactogenicity and safety of 2 doses of an adjuvanted herpes zoster subunit vaccine administered 2, 6 or 12 months apart in older adults: results of a phase III, randomized, open-label, multicenter study. Vaccine. 2018;36(1):148-154.

50. Godeaux O, Kovac M, Shu D, et al. Immunogenicity and safety of an adjuvanted herpes zoster subunit candidate vaccine in adults $\geq 50$ years of age with a prior history of herpes zoster: a phase III, non-randomized, openlabel clinical trial. Hum Vaccin Immunother. 2017;13(5):1051-1058.

51. Grupping K, Campora L, Douha M, et al. Immunogenicity and safety of the $\mathrm{HZ} / \mathrm{su}$ adjuvanted herpes zoster subunit vaccine in adults previously vaccinated with a live attenuated herpes zoster vaccine. J Infect Dis. 2017;216(11):1343-1351.

52. Vink P, Shiramoto M, Ogawa M, et al. Safety and immunogenicity of a herpes zoster subunit vaccine in Japanese population aged $\geq 50$ years when administered subcutaneously vs. intramuscularly. Hum Vaccin Immunother. 2017;13(3):574-578

53. Cunningham AL. The herpes zoster subunit vaccine. Expert Opin Biol Ther. 2016;16(2):265-271.

54. Berkowitz EM, Moyle G, Stellbrink HJ, et al; Zoster-015 HZ/su Study Group. Safety and immunogenicity of an adjuvanted herpes zoster subunit candidate vaccine in HIV-infected adults: a phase 1/2 a randomized, placebo-controlled study. J Infect Dis. 2015;211(8):1279-1287.

55. Stadtmauer EA, Sullivan KM, Marty FM, et al. A phase $1 / 2$ study of an adjuvanted varicella-zoster virus subunit vaccine in autologous hematopoietic cell transplant recipients. Blood. 2014;124(19):2921-2929.

56. [webpage on the Internet]. Study to Evaluate Efficacy, Safety, and Immunogenicity of GlaxoSmithKline (GSK) Biologicals'Herpes Zoster Vaccine GSK1437173A. Available from: https:/www.clinicaltrials.gov/ ct2/show/results/NCT01610414?term=01610414\&rank=1\&sect=X012 56\#all. NLM identifier: NCT01610414 Accessed July 19, 2018.

57. Schwarz TF, Aggarwal N, Moeckesch B, et al. Immunogenicity and Safety of an adjuvanted herpes zoster subunit vaccine coadministered with seasonal influenza vaccine in adults aged 50 years or older. $J$ Infect Dis. 2017;216(11):1352-1361.

58. Food and Drugs Administration (FDA). Shingrix Highlights of Prescribing Information. Available from: https:/www.fda.gov/downloads/ biologicsbloodvaccines/vaccines/approvedproducts/ucm581605.pdf. Accessed May 14, 2018.
59. Cunningham AL, Heineman T. Vaccine profile of herpes zoster (HZ/su) subunit vaccine. Expert Rev Vaccines. 2017;16(7):661-670.

60. European Medicines Agency (EMA). SPC Shingrix. Available from: http://www.ema.europa.eu/docs/it_IT/document_library/EPAR_-_Product_Information/human/004336/WC500246550.pdf. Accessed May 22, 2018.

61. Kovac M, Lal H, Cunningham AL, et al; ZOE-50/70 Study Group. Complications of herpes zoster in immunocompetent older adults: incidence in vaccine and placebo groups in two large phase 3 trials. Vaccine. 2018;36(12):1537-1541.

62. Curran D, van Oorschot D, Varghese L, et al. Assessment of the potential public health impact of herpes zoster vaccination in Germany. Hum Vaccin Immunother. 2017;13(10):2213-2221.

63. Watanabe D, Mizukami A, Holl K, et al. The potential public health impact of herpes zoster vaccination of people aged $\geq 50$ years in Japan: results of a Markov model analysis. Dermatol Ther (Heidelb). 2018;8:269-284

64. Le P, Rothberg MB. Cost-effectiveness of the adjuvanted herpes zoster subunit vaccine in older adults. JAMA Intern Med. 2018;178(2) 248-258.

65. Dooling KL, Guo A, Patel M, et al. Recommendations of the advisory committee on immunization practices for use of herpes zoster vaccines. MMWR Morb Mortal Wkly Rep. 2018;67(3):103-108.

66. Maréchal $\mathrm{C}$, Lal H, Poder A, et al. Immunogenicity and safety of the adjuvanted recombinant zoster vaccine co-administered with the 23 -valent pneumococcal polysaccharide vaccine in adults $\geq 50$ years of age: a randomized trial. Vaccine. 2018;36(29):4278-4286.

67. [webpage on the Internet]. Immunogenicity and Safety of GlaxoSmithKline (GSK) Biologicals' Herpes Zoster Subunit (HZ/su) Vaccine in Adults 18 Years of Age or Older With Renal Transplant. Available from: https:/clinicaltrials.gov/ct2/show/NCT02058589?term=NCT0 2058589\&rank=1. NLM identifier: NCT02058589 Accessed May 23, 2018.

68. [webpage on the Internet]. Immunogenicity and Safety Study of GlaxoSmithKline (GSK) Biologicals'Herpes Zoster (HZ/su) Vaccine in Adults With Solid Tumours Receiving Chemotherapy. Available from: https:// clinicaltrials.gov/ct2/show/NCT01798056?term=NCT01798056\&r ank=1. NLM identifier: NCT01798056 Accessed May 23, 2018.

69. [webpage on the Internet]. Study to Assess the Safety and Immunogenicity of GlaxoSmithKline (GSK) Biologicals'Herpes Zoster Subunit (HZ) su) Vaccine in Adults Aged 18 Years and Older With Blood Cancers. Available from: https:/clinicaltrials.gov/ct2/show/NCT01767467?ter $\mathrm{m}=\mathrm{NCT} 01767467 \&$ rank=1. NLM identifier: NCT01767467 Accessed May 23, 2018.
Infection and Drug Resistance

\section{Publish your work in this journal}

Infection and Drug Resistance is an international, peer-reviewed openaccess journal that focuses on the optimal treatment of infection (bacterial, fungal and viral) and the development and institution of preventive strategies to minimize the development and spread of resistance. The journal is specifically concerned with the epidemiology of antibiotic

\section{Dovepress}

resistance and the mechanisms of resistance development and diffusion in both hospitals and the community. The manuscript management system is completely online and includes a very quick and fair peerreview system, which is all easy to use. Visit http://www.dovepress.com/ testimonials.php to read real quotes from published authors. 